\title{
Effect of Endotracheal Suctioning on Infants Born through Meconium-Stained Amniotic Fluid: A Meta-Analysis
}

\author{
Qing Wei, BS $1,2,3,4$ Wenjing Chen, BS ${ }^{1,2,3,4}$ Qian Liang, MS $^{1,2,3,4}$ Shurong Song, BS $1,2,3,4$
}

Jia Li, MS ${ }^{1,2,3,4}$

\footnotetext{
${ }^{1}$ Department of Obstetrics, The Third Central Hospital of Tianjin, Hedong District, Tianjin, China

2 Tianjin Key Laboratory of Extracorporeal Life Support for Critical Diseases, Tianjin, China

${ }^{3}$ Department of obstetrics, Artificial Cell Engineering Technology Research Center, Tianjin, China

${ }^{4}$ Tianjin Institute of Hepatobiliary Disease, Tianjin, China
}

\begin{abstract}
Address for correspondence Qian Liang, MS, Department of Obstetrics, The Third Central Hospital of Tianjin, 83 jintang Road, Hedong District, Tianjin 300170, China, Tianjin Key Laboratory of Extracorporeal Life Support for Critical Diseases, Tianjin, China, Artificial Cell Engineering Technology Research Center, Tianjin, China, Tianjin Institute of Hepatobiliary Disease, Tianjin, China (e-mail: xixilq2009@163.com).
\end{abstract}

Am J Perinatol

\begin{abstract}
Keywords

- meconium aspiration syndrome

- suction

- odds ratios

- endotracheal

- meta-analysis

Objective Meconium is a common finding in amniotic fluid and placental specimens, particularly in term and post-term pregnancies. The objective of this paper was to perform a meta-analysis to examine the impact of endotracheal suctioning on the occurrence of meconium aspiration syndrome (MAS), mortality, and complications.

Study Design PubMed, EMBASE, and the Cochrane library were systematically searched for comparative studies. Odds ratios (ORs), weighted mean differences (WMDs), and corresponding 95\% confidence intervals (Cls) were used to compare the outcomes.

Results Twelve studies were included in the meta-analysis. There were no significant impacts of endotracheal suctioning on the occurrence of MAS $(\mathrm{OR}=3.05,95 \% \mathrm{Cl}$ : $0.48-19.56)$, mortality $(\mathrm{OR}=1.25,95 \% \mathrm{Cl}: 0.35-4.44)$, the need for mechanical ventilation ( $\mathrm{OR}=4.20,95 \% \mathrm{Cl}: 0.32-54.72$ ), the occurrence of pneumothorax (OR $=0.99,95 \% \mathrm{Cl}: 0.34-2.85)$, persistent pulmonary hypertension of the newborn (PPHN), (OR =1.31, 95\% Cl: 0.58-2.98), hypoxic-ischemic encephalopathy (HIE) (OR $=0.82,95 \% \mathrm{Cl}: 0.52-1.30$ ), and length of stay (WMD $=-0.11,95 \% \mathrm{Cl}:-0.99-0.77)$. Conclusion Routine endotracheal suctioning at birth is not useful in preventing MAS, mortality, mechanical ventilation, PPHN, HIE, and prolonged length of stay in neonates born through MSAF.
\end{abstract}

Key Points

- Routine suctioning is not recommended for newborns.

- Endotracheal aspiration is not beneficial for MAS.

- Future research may focus on selected neonates.

I These authors contributed equally to this work.

received

September 7, 2021

accepted after revision

November 29, 2021 (c) 2022. Thieme. All rights reserved.

Thieme Medical Publishers, Inc., 333 Seventh Avenue, 18th Floor, New York, NY 10001, USA
DOI https://doi.org/ 10.1055/s-0041-1741034. ISSN 0735-1631. 
Meconium is a common finding in amniotic fluid and placental specimens, particularly in term and post-term pregnancies. $^{1-4}$ The most important consequence of meconiumstained amniotic fluid (MSAF) is the meconium aspiration syndrome (MAS). ${ }^{1-3,5}$ The MAS is a condition marked by respiratory distress in a neonate after delivery through MSAF. $^{1-3,5}$ The aspiration of meconium usually occurs in utero but may also happen after delivery. ${ }^{1-3,5}$ Factors that increase the possibility of MAS include intrauterine growth restriction, delivery at $>41$ weeks of gestation, heavy MSAF, the presence of meconium below the vocal cords, and fetal heart rate abnormalities during labor. ${ }^{2,3,6,7}$ Symptoms of MAS usually develop within 15 minutes of birth but may take up to 12 hours. Neonates usually present with respiratory distress, including tachypnea, cyanosis, grunting, nasal flaring, and retractions. ${ }^{1-3}$ If fetal distress is present, neonates may also have symptoms of neonatal depression, including bradycardia, decreased respiratory effort, and reduced muscle tone. ${ }^{1-3}$ At least $5 \%$ of infants born through MSAF develop MAS. ${ }^{1-3,5,8,9}$ MAS continues to be a threat to many newborns throughout the world, with a fatality rate of 5 to $40 \%$, in addition to short- and long-term pulmonary and neurological developmental sequelae. 2,3,5,10

Universal intrapartum suction of infants with MSAF and postnatal suction of vigorous infants have been used in an attempt to decrease the incidence and severity of MAS by clearing the airway, but the practice of endotracheal suctioning of meconium-stained non-vigorous newborns has been questioned due to procedure-related harms and uncertain benefits. The current guidelines from the American College of Obstetricians and Gynecologists (ACOG) do not recommend the routine intrapartum suctioning of all newborns with MSAF; gentle suctioning can be done in vigorous neonates, and suctioning is not recommended for nonvigorous newborns, but endotracheal suctioning can be considered if breathing is obstructed by a meconium plug. ${ }^{11-13}$ Nevertheless, available studies report conflicting results with studies supporting endotracheal suctioning, ${ }^{14,15}$ supporting no endotracheal suctioning, ${ }^{16,17}$ and with negative results. ${ }^{7,18-22}$ When this meta-analysis was conducted, there was no meta-analysis on the topic. Still, one meta-analysis was published in the meantime, suggesting no benefit of endotracheal suctioning in non-vigorous newborns. $^{23}$

Therefore, we performed a meta-analysis and systemic review to examine the impact of endotracheal suctioning on MAS occurrence, mortality, and complications in all infants born through MSAF. The results could shed some light on the possible benefits of this practice.

\section{Materials and Methods}

\section{Literature Search}

This systematic review and meta-analysis were performed according to the Preferred Reporting Items for Systematic Reviews and Meta-Analyses guidelines ${ }^{24}$ and the PICO methodology. ${ }^{25}$ PubMed, EMBASE, and the Cochrane library were systematically searched for studies published up to Novem- ber 2019. The search strategies are presented in - Supplemental Table 1.

The eligibility criteria were as follows: (1) population: newborn infants born through MSAF; (2) intervention: endotracheal suctioning; (3) control: without endotracheal suctioning; (4) study types: cohort study and randomized control trial (RCT); and (5) language: limited to English.

\section{Search Strategy}

We performed a systematic search from the PubMed, Embase, and Cochrane library databases for available papers published up to November 2019 using the Mesh terms "MAS," and "Suction," as well as relevant keywords. The reference lists of the identified papers were reviewed to find additional eligible studies.

\section{Data Extraction and Quality Assessment}

The selection and inclusion of studies were performed in two stages by two independent reviewers (Q.W. and Q.L.). This included the analysis of the titles and abstracts, followed by the full texts. Disagreements were resolved by discussion with a third reviewer (W.C.).

Data including authors, publication year, study design, gestational maturity, birth weight, vigorous/non-vigorous babies, MAS, mortality, pneumothorax, persistent pulmonary hypertension of the newborn (PPHN), mechanical ventilation, and hypoxic-ischemic encephalopathy (HIE) were extracted from the papers.

The RCTs were evaluated according to the Cochrane risk bias tool. ${ }^{26}$ The observational studies were evaluated according to the Newcastle-Ottawa Scale (NOS). ${ }^{27}$

\section{Statistical Analysis}

All analyses were performed using the STATA SE 14.0 software (StataCorp, College Station, TX, USA). Odds ratios (ORs), weighted mean differences (WMDs), and the corresponding 95\% confidence intervals (CIs) were used to compare the outcomes. Statistical heterogeneity among the included studies was calculated using Cochran's Q-test and the $I^{2}$ index ( $I^{2}>50 \%$ indicated high heterogeneity). The randomeffects model was used when high heterogeneity was present among studies; otherwise, the fixed-effects model was applied. $p$-Values $<0.05$ were considered statistically significant. Potential publication bias was assessed using funnel plots and Egger's and Begg tests. ${ }^{26}$

\section{Results}

\section{Literature Search}

The database search identified 479 records, and 318 records were left after removing the duplicates. After screening the titles and abstracts, 55 full-text articles were assessed for eligibility. Finally, 12 articles were included in the meta-analysis (-Supplemental Fig. 1 and -Supplemental Table 1). -Supplemental Table 2 summarizes the included papers published between 1975 and 2019. There were seven RCTs and five cohort studies. The sample sizes ranged from 122 to 11,344 neonates, for a total of 16,828. - Supplemental Table 2 
A

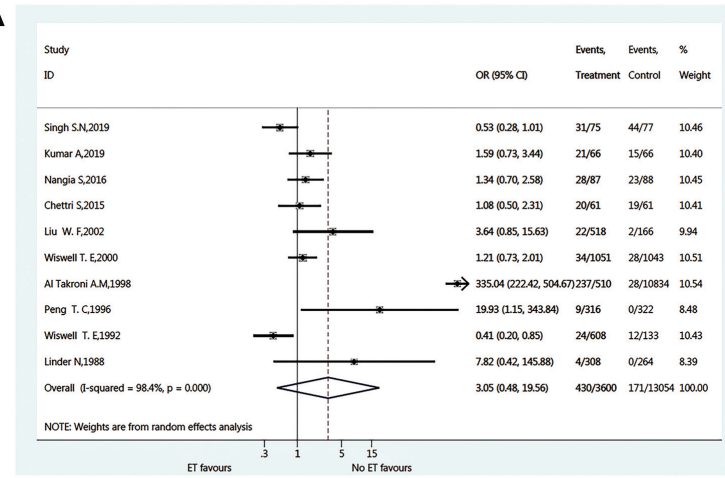

B

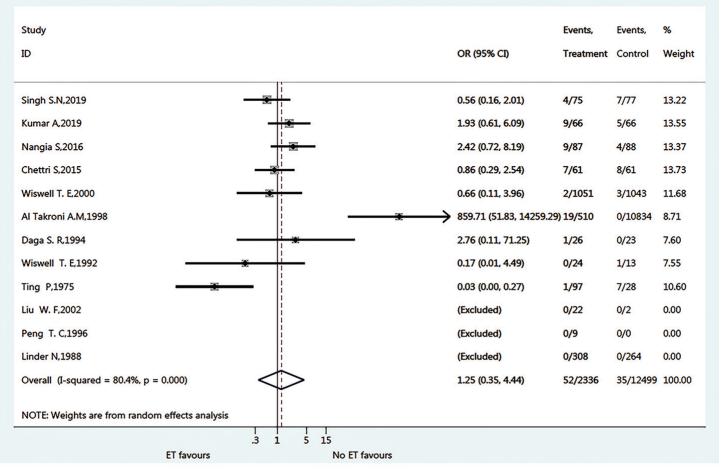

C

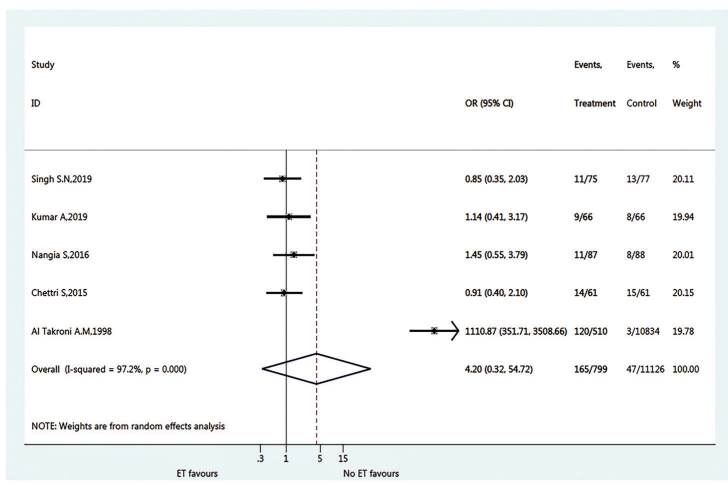

Fig. 1 (A) Forest plot of MAS. (B) Forest plot of mortality. (C) Forest plot of mechanical ventilation.

presents the bias analysis of the RCTs; except for the RCT by Singh et al, $^{14}$ all other RCTs ${ }^{18-22,28}$ had low probabilities of biases. - Supplemental Tables 3-4 present the NOS evaluation of the cohort studies. Among the cohort studies, three $16,17,29$ scored seven stars on the NOS, while two ${ }^{7,15}$ scored eight stars.

\section{MAS}

Ten studies ${ }^{7,14-22}$ could be included in the MAS analysis. The meta-analysis showed no significant impact of endotracheal suctioning on the occurrence of MAS ( $\mathrm{OR}=3.05,95 \% \mathrm{CI}$ : 0.48-19.56, $p=0.239$ ) ( - Fig. 1A). Heterogeneity was observed, and the random-effect model was used $\left(I^{2}=98.4 \%\right.$, $p<0.001$ ). The subgroup analyses showed similar results (all $p>0.05$ ) ( - Table 1 ). The sensitivity analysis indicated that the study by $\mathrm{Al}$ Takroni et $\mathrm{al}^{16}$ introduced heterogeneity (-Supplemental Fig. 2A). After excluding that study, there was no significant impact of endotracheal suctioning on the occurrence of MAS (OR=1.16, 95\% CI: $0.71-1.88, p=0.554$ )

\begin{tabular}{|llllllll|}
\hline \multicolumn{2}{|l}{ Table 1 Subgroup analysis of MAS } & & & \\
\hline MAS & & Treatment & Control & & & \\
& $n$ & Event/total (\%) & Event/total (\%) & OR $(95 \%$ CI) & $P$ & $I^{2}$ & $P_{\text {heterogeneity }}$ \\
MAS in all & 10 & $430 / 3,600(11.9)$ & $171 / 13,054(1.3)$ & $3.051(0.476,19.565)$ & 0.239 & 98.4 & $<0.001$ \\
RCT & 6 & $138 / 1,648(8.4)$ & $129 / 1,599(8.1)$ & $1.105(0.751,1.626)$ & 0.611 & 38 & 0.153 \\
Observational study & 4 & $292 / 1,952(15.0)$ & $42 / 11,455(0.4)$ & $9.952(0.115,860.352)$ & 0.313 & 99 & $<0.001$ \\
Aisa & 5 & $337 / 799(42.2)$ & $129 / 11,126(1.2)$ & $3.347(0.170,65.949)$ & 0.427 & 99.1 & $<0.001$ \\
America & 5 & $93 / 2,801(3.3)$ & $42 / 1,928(2.2)$ & $1.767(0.601,5.196)$ & 0.301 & 76 & 0.002 \\
Non-vigorous & 4 & $100 / 289(34.6)$ & $101 / 292(34.6)$ & $1.027(0.625,1.687)$ & 0.916 & 49.5 & 0.114 \\
Vigorous & 4 & $69 / 2,193(3.1)$ & $30 / 1,795(1.7)$ & $3.116(0.898,10.814)$ & 0.073 & 57.5 & 0.07 \\
Unclear & 2 & $261 / 1,118(23.3)$ & $40 / 10,967(0.4)$ & $3.051(0.476,19.565)$ & 0.488 & 99.6 & $<0.001$ \\
After 2010 & 4 & $100 / 289(34.6)$ & $101 / 292(34.6)$ & $1.027(0.625,1.687)$ & 0.916 & 49.5 & 0.114 \\
Before 2010 & 6 & $330 / 3,311(10.0)$ & $70 / 12,762(0.5)$ & $6.618(0.321,136.427)$ & 0.221 & 98.8 & $<0.001$ \\
Sample size $<200$ & 4 & $100 / 289(34.6)$ & $101 / 292(34.6)$ & $1.027(0.625,1.687)$ & 0.916 & 49.5 & 0.114 \\
Sample size $>200$ & 6 & $330 / 3,311(10.0)$ & $70 / 12,762(0.5)$ & $6.618(0.321,136.427)$ & 0.221 & 98.8 & $<0.001$ \\
\hline
\end{tabular}

Abbreviations: $\mathrm{Cl}$, confidence interval; MAS, meconium aspiration syndrome; OR, odds ratio; RCT, randomized control trial. 


\begin{tabular}{|c|c|c|c|c|c|c|c|}
\hline \multirow[t]{2}{*}{ Mortality } & \multirow[b]{2}{*}{$n$} & \multirow{2}{*}{$\begin{array}{l}\text { Treatment } \\
\text { Event/total (\%) }\end{array}$} & \multicolumn{5}{|l|}{ Control } \\
\hline & & & Event/total (\%) & OR $(95 \% \mathrm{Cl})$ & $p$ & $r^{2}$ & $P_{\text {heterogeneity }}$ \\
\hline Mortality in all & 12 & $52 / 2,336(2.2)$ & $35 / 12,499(0.3)$ & $1.254(0.354,4.442)$ & 0.725 & 80.4 & $<0.001$ \\
\hline RCT & 7 & $32 / 1,674(1.9)$ & $27 / 1,622(1.7)$ & $1.190(0.686,2.062)$ & 0.536 & 0 & 0.503 \\
\hline Observational study & 5 & $20 / 662(3.0)$ & $8 / 10,877(0.1)$ & $1.630(0.002,1,237.168)$ & 0.885 & 94.4 & $<0.001$ \\
\hline Aisa & 6 & 49/825 (5.9) & $24 / 11,149(0.2)$ & $2.952(0.695,12.542)$ & 0.142 & 82.1 & $<0.001$ \\
\hline America & 6 & $3 / 1,511(0.2)$ & $11 / 12,499(0.1)$ & $0.161(0.021,1.230)$ & 0.078 & 56.5 & 0.1 \\
\hline Non-vigorous & 5 & $30 / 315(9.5)$ & $24 / 315(7.6)$ & $1.265(0.710,2.254)$ & 0.425 & 0 & 0.423 \\
\hline Vigorous & 4 & $2 / 1,390(0.1)$ & $3 / 1,309(0.2)$ & $0.661(0.110,3.964)$ & 0.65 & - & - \\
\hline Unclear & 3 & 20/631 (3.2) & $8 / 10,875(0.1)$ & $1.630(0.002,1,237.168)$ & 0.725 & 94.4 & $<0.001$ \\
\hline After 2010 & 4 & 29/289 (10.0) & $24 / 292(8.2)$ & $1.233(0.645,2.357)$ & 0.527 & 17.7 & 0.302 \\
\hline Before 2010 & 8 & $23 / 2,047(1.1)$ & $11 / 12,207(0.1)$ & $1.464(0.046,46.945)$ & 0.829 & 89.3 & $<0.001$ \\
\hline Sample size $<200$ & 6 & $31 / 412(7.5)$ & $31 / 343(9.0)$ & $0.826(0.297,2.302)$ & 0.715 & 66 & 0.012 \\
\hline Sample size $>200$ & 6 & $21 / 1,924(1.1)$ & $4 / 12,156(0.03)$ & $4.576(0.028,748.847)$ & 0.559 & 91.5 & $<0.001$ \\
\hline
\end{tabular}

Abbreviations: $\mathrm{Cl}$, confidence interval; OR, odds ratio; $\mathrm{RCT}$, randomized control trial.

(-Supplemental Fig. 2B). Meta-regression showed that the publication year was not associated with the occurrence of MAS ( $p=0.277)$ (-Supplemental Fig. 2C).

\section{Mortality}

Nine studies [14-16,18-21,25,26] were included in the mortality analysis. The meta-analysis showed no significant impact of endotracheal suctioning on mortality $(\mathrm{OR}=1.25$, 95\% CI: 0.35-4.44, $p=0.725$ ) ( - Fig. 1B). Heterogeneity was observed, and the random-effect model was used $\left(I^{2}=80.4 \%\right.$, $p<0.001$ ). The subgroup analyses showed similar results (all $p>0.05)$ ( - Table 2). The sensitivity analysis indicated that $\mathrm{Al}$ Takroni et al. [16] introduced heterogeneity ( - Supplemental Fig. 3A). After excluding that study, there was no significant impact of endotracheal suctioning on mortality $(\mathrm{OR}=0.75$, 95\% CI: 032-1.77, $p=0.513$ ) (-supplemental Fig. 3B).

\section{Mechanical Ventilation}

Five studies $14,16,18,19,22$ could be included in the analysis of mechanical ventilation. The meta-analysis showed no significant impact of endotracheal suctioning on the need for mechanical ventilation $(\mathrm{OR}=4.20,95 \% \mathrm{CI}$ : $0.32-54.72$, $p=0.273$ ) (-Fig. 1C). Heterogeneity was observed, and the random-effect model was used $\left(I^{2}=97.2 \%, p<0.001\right)$. After excluding the study by $\mathrm{Al}$ Takroni et al [16], similar results were still observed ( $\mathrm{OR}=1.04,95 \% \mathrm{CI}: 0.66-1.64, p=0.869)$ but without heterogeneity $\left(I^{2}=0 \%\right)$.

\section{Other Outcomes}

-Fig. 2A to D show that endotracheal suctioning does not affect the occurrence of pneumothorax $(\mathrm{OR}=0.99,95 \% \mathrm{CI}$ : $0.34-2.85, p=0.979 ; I^{2}=0 \%, p=0.872$ ) [14,19,20,25], PPHN $\left(\mathrm{OR}=1.31,95 \% \mathrm{CI}: 0.58-2.98, p=0.513 ; I^{2}=0 \%, p=0.598\right)$ $[14,18,20]$, HIE $(\mathrm{OR}=0.82,95 \% \mathrm{CI}: 0.52-1.30, p=0.405$; $\left.I^{2}=12.9 \%, p=0.317\right)[14,19,25]$, and the length of stay (WMD $\left.=-0.11, \quad 95 \% \quad \mathrm{CI}: \quad-0.99-0.77 ; \quad I^{2}=72.8 \%, \quad p=0.025\right)$ $[14,18,19]$.

\section{Publication Bias}

The funnel plots revealed some publication bias regarding MAS (-Supplemental Fig. 4A) and mortality (-Supplemental Fig. 4B), but there were no significant differences in Egger's test $\left(P_{\text {MAS }}=0.348, P_{\text {mortality }}=0.828\right)$ and Begg's test $\left(P_{\text {MAS }}=0.107, P_{\text {mortality }}=0.754\right)$.

\section{Discussion}

Endotracheal suctioning of meconium-stained newborns has been questioned due to procedure-related harms and uncertain benefits. ${ }^{7,11-22}$ Therefore, the present meta-analysis aimed to examine the impact of endotracheal suctioning on MAS occurrence, mortality, and complications. The results strongly suggest that routine endotracheal suctioning at birth is not useful in preventing MAS, mortality, mechanical ventilation, PPHN, HIE, and prolonged length of stay in neonates born through MSAF.

In the present meta-analysis, $\mathrm{Al}$ Takroni et $\mathrm{al}^{16}$ systematically introduced significant heterogeneity in all analyses. This was a retrospective cohort study of 11,344 births at a hospital where the babies born through MSAF undergo intrapartum endotracheal suctioning, followed by intubation for asphyxiated babies and observation for vigorous ones. There was no distinction based on gestational age, while nine studies included term and post-term neonates, two studies did not mention the gestational age., ${ }^{75}$ Even though delivery through MSAF is more likely to occur in neonates at term or post-term, ${ }^{2,3,6,7}$ there is a possibility that the study by Al Takroni et $\mathrm{al}^{16}$ included pre-term neonates. In addition, the incidence of delivery through MSAF was elevated, at $13.3 \%$.

In the present meta-analysis of 16,828 newborns (12 studies), no benefit of endotracheal suctioning was observed in all newborns, irrespective of status. This is supported by a meta-analysis published while the present one was being conducted. In that recent meta-analysis of 581 newborns 
A

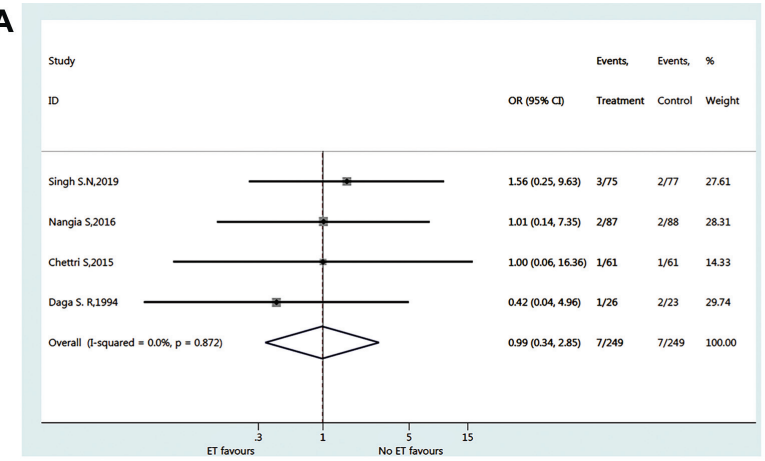

C

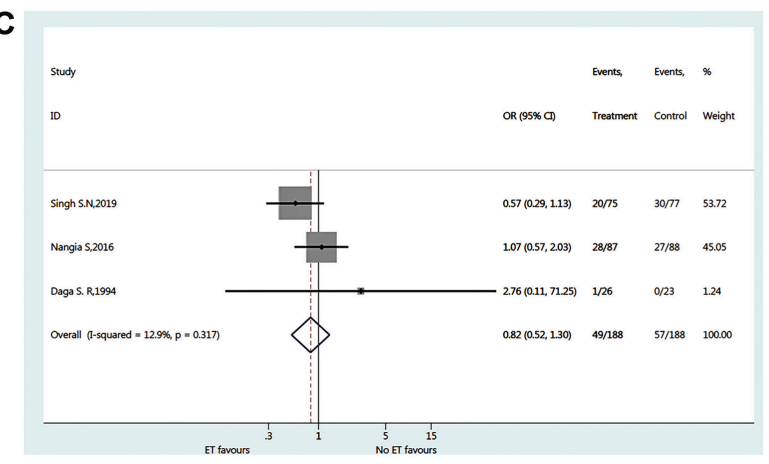

B

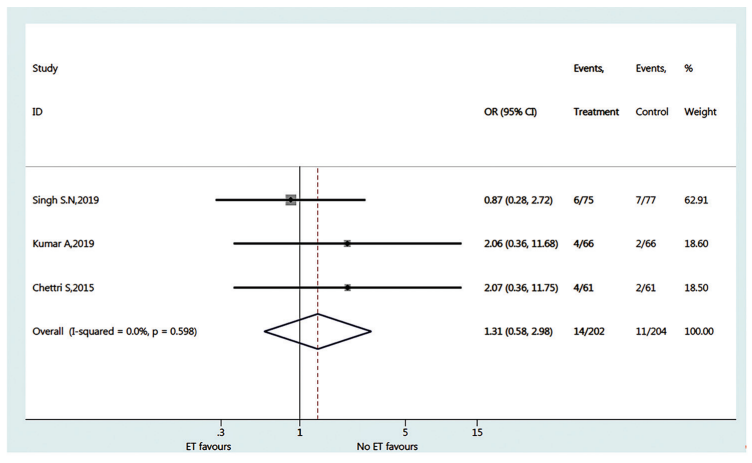

D

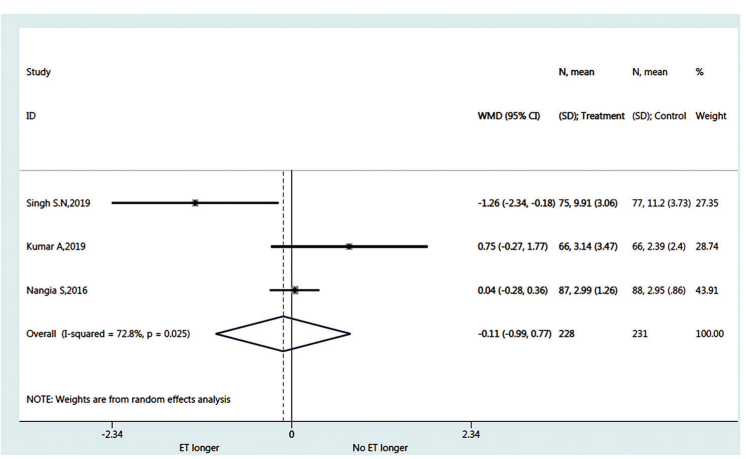

Fig. 2 Forest plot of (A) pneumothorax; (B) persistent pulmonary hypertension of newborn; (C) hypoxic-ischemic encephalopathy; and (D) length of stay.

(four studies), endotracheal suctioning in non-vigorous newborns apparently did not improve neonatal outcomes. ${ }^{23}$ Still, we used different inclusion criteria. Indeed, Phattraprayoon et $\mathrm{al}^{23}$ included only four studies, while the present metaanalysis included 12 . Using too stringent selection criteria carries the risk of decreasing the generalizability of the conclusions. The populations were different, with non-vigorous infants in Phattraprayoon et $\mathrm{al}^{23}$ and infants born through MSAF in the present study. The outcomes were also different, the present meta-analysis examining MAS, mortality, the need for mechanical ventilation, the occurrence of pneumothorax, PPHN, HIE, and length of stay, while the previous meta-analysis examined MAS, pneumothorax, PPHN, secondary pneumonia, need for respiratory support, duration of mechanical ventilation, initial resuscitation, and others including shock, perinatal asphyxia, convulsions, neonatal mortality, blood culture-positive sepsis, and duration of hospital stay. Examining too many outcomes using a small number of studies increases the risk of misleading conclusions. In addition, because of the small number of studies included, subgroup analyses were very limited in Phattraprayoon et al. ${ }^{23}$ Still, they reached a conclusion similar to the present meta-analysis. The meta-analysis by Phattraprayoon et $\mathrm{al}^{23}$ demonstrated no benefits in nonvigorous infants, while the present meta-analysis demonstrated no benefits in all infants born through MSAF.

The failure of endotracheal suctioning in improving the outcomes of the newborn might include the occurrence of meconium aspiration in utero, the migration of the meconium to the distant airways, and the impossibility of removing the meconium from those small airways. ${ }^{30}$ In addition, even if some meconium could be aspirated from the distal airways, there is still a high possibility of some of them remaining plugged. ${ }^{18}$ In addition to the mechanical concept regarding the failure to aspirate the meconium, MAS induces pulmonary and systemic pathophysiological changes that include inactivation of surfactant, ${ }^{31}$ pulmonary hypertension, ${ }^{32}$ and activation of immunity, ${ }^{33}$ which would lead to poor outcomes even if all meconium were removed. Therefore, the current guidelines from the ACOG do not recommend the routine intrapartum suctioning of all newborns with MSAF. ${ }^{11}$ Instead, they recommend that gentle suctioning can be done in vigorous neonates but not in non-vigorous newborns and that endotracheal suctioning can be considered if breathing is obstructed by a meconium plug. ${ }^{11}$ Those recommendations are supported by the American Heart Association (AHA) ${ }^{12}$ and the 2015 International Consensus on Cardiopulmonary Resuscitation and Emergency Cardiovascular Care Science. ${ }^{13}$ Nevertheless, endotracheal suctioning might still be required in selected neonates. Indeed, despite that the changes in practice following Neonatal Resuscitation Program (NRP) have not increased the reported cases of MAS, an increase in neonatal intensive care unit admissions for respiratory distress has been observed, with higher rates of requirement for mechanical ventilation, oxygen, and surfactants. ${ }^{34}$ In addition, the main issue with endotracheal suctioning is the possible delay in the resuscitation of already compromised neonates with the possibility of asphyxia injuries. ${ }^{35}$ The NRP guidelines state that positive pressure ventilation must be performed if required after endotracheal suction and evaluation. ${ }^{36}$ Such forced ventilation might alleviate the impact of asphyxia 
despite a delay due to endotracheal suctioning. Therefore, future studies should focus on selecting the neonates who would benefit the most from endotracheal suctioning. Nevertheless, the most recent studies suggest no benefit, no harm either, of endotracheal suctioning. ${ }^{14,18,22}$ Nevertheless, those studies were performed in selected populations. Kumar et $\mathrm{al}^{18}$ and Singh et $\mathrm{al}^{14}$ showed that endotracheal suctioning did not prevent MSAF in non-vigorous neonates of $^{3} 34$ weeks of gestation. Nangia et $\mathrm{al}^{22}$ reached a similar conclusion in non-vigorous newborns of ${ }^{3} 37$ weeks of gestation. Therefore, it is possible that endotracheal suctioning only benefits very selected newborns. Future studies could seek to identify those newborns.

This study had limitations. Although several studies were initially identified, the strict inclusion criteria resulted in only 12 studies being included. Since a preliminary search revealed small numbers of RCTs and cohort studies, both study types were included, resulting in biases based on the analysis of the different data types. In addition, heterogeneity was high, even after excluding the study by Al Takroni et al. ${ }^{16} \mathrm{~A}$ better level of evidence could still be necessary to reach firm conclusions on endotracheal suctioning in neonates born through MSAF.

\section{Conclusions}

Routine endotracheal suctioning at birth shows no obvious benefit for preventing MAS, mortality, mechanical ventilation, PPHN, HIE, and prolonged length of stay in neonates born through MSAF.

\section{Data Availability Statement}

The data used to support the findings of this study are available from the corresponding author upon request.

\section{Authors' Contributions}

Q.W. substantially contributed to the conception or design, acquisition, analysis, and interpretation of data. WC contributed to acquisition, analysis, interpretation of data, and drafted the manuscript for important content. QL critically revised the manuscript for important intellectual content. SS gave final approval. JL contributed to acquisition, analysis, and interpretation of data.

\section{Funding \\ None. \\ Conflict of Interest \\ None declared. \\ Acknowledgments \\ Not applicable.}

\section{References}

1 Walsh MC, Fanaroff JM. Meconium stained fluid: approach to the mother and the baby. Clin Perinatol 2007;34(04):653-665

2 Bhat R, Vidyasagar D. Delivery room management of meconiumstained infant. Clin Perinatol 2012;39(04):817-831
3 Yeh T. Core concepts: meconium aspiration syndrome: pathogenesis and current management. Neoreviews 2010;11:e503-e512

4 Asad A, Bhat R. Pharmacotherapy for meconium aspiration. J Perinatol Official J Calif Perinatal Assoc 2008;28(Suppl 3): S72-S78

5 Chettri S, Bhat BV, Adhisivam B. Current Concepts in the Management of Meconium Aspiration Syndrome. Indian J Pediatr 2016;83 (10):1125-1130

$6 \mathrm{Xu} \mathrm{H}$, Wei S, Fraser WD. Obstetric approaches to the prevention of meconium aspiration syndrome. J Perinatol Official J Calif Perinatal Assoc 2008;28(Suppl 3):S14-S18

7 Liu WF, Harrington T. Delivery room risk factors for meconium aspiration syndrome. Am J Perinatol 2002;19(07):367-378

8 Fischer C, Rybakowski C, Ferdynus C, Sagot P, Gouyon JB. A population-based study of meconium aspiration syndrome in neonates born between 37 and 43 weeks of gestation. Int J Pediatr 2012;2012:321545

9 Dargaville PA, Copnell BAustralian and New Zealand Neonatal Network. The epidemiology of meconium aspiration syndrome: incidence, risk factors, therapies, and outcome. Pediatrics 2006; 117(05):1712-1721

10 Dargaville PA. Respiratory support in meconium aspiration syndrome: a practical guide. Int J Pediatr 2012;2012:965159

11 Committee Opinion No. Committee opinion no.689 summary: delivery of a newborn with meconium-stained amniotic fluid. Obstet Gynecol 2017;129(03):593-594

12 Wyckoff MH, Aziz K, Escobedo MB, et al. Part 13: neonatal resuscitation: 2015 American Heart Association Guidelines Update for Cardiopulmonary Resuscitation and Emergency Cardiovascular Care (Reprint). Pediatrics 2015;136(Suppl 2):S196-S218

13 Perlman JM, Wyllie J, Kattwinkel J, et al; Neonatal Resuscitation Chapter Collaborators. Part 7: neonatal resuscitation: 2015 International Consensus on Cardiopulmonary Resuscitation and Emergency Cardiovascular Care Science With Treatment Recommendations. Circulation 2015;132(16, Suppl 1)S204-S241

14 Singh SN, Saxena S, Bhriguvanshi A, Kumar MCS. Effect of endotracheal suctioning just after birth in non-vigorous infants born through meconium stained amniotic fluid: a randomized controlled trial. Clin Epidemiol Glob Health 2019;7:165-170

15 Wiswell TE, Henley MA. Intratracheal suctioning, systemic infection, and the meconium aspiration syndrome. Pediatrics 1992;89 (02):203-206

16 Al Takroni AM, Parvathi CK, Mendis KB, Hassan S, Reddy I, Kudair HA. Selective tracheal suctioning to prevent meconium aspiration syndrome. Int J Gynaecol Obstet 1998;63(03):259-263

17 Peng TC, Gutcher GR, Van Dorsten JP. A selective aggressive approach to the neonate exposed to meconium-stained amniotic fluid. Am J Obstet Gynecol 1996;175(02):296-301, discussion 301-303

18 Kumar A, Kumar P, Basu S. Endotracheal suctioning for prevention of meconium aspiration syndrome: a randomized controlled trial. Eur J Pediatr 2019;178(12):1825-1832

19 Chettri S, Adhisivam B, Bhat BV. Endotracheal suction for nonvigorous neonates born through meconium stained amniotic fluid: a randomized controlled trial. J Pediatr 2015;166(05): 1208-1213.e1

20 Wiswell TE, Gannon CM, Jacob J, et al. Delivery room management of the apparently vigorous meconium-stained neonate: results of the multicenter, international collaborative trial. Pediatrics 2000; 105(1 Pt 1):1-7

21 Linder N, Aranda JV, Tsur M, et al. Need for endotracheal intubation and suction in meconium-stained neonates. J Pediatr 1988; 112(04):613-615

22 Nangia S, Sunder S, Biswas R, Saili A. Endotracheal suction in term non-vigorous meconium stained neonates-a pilot study. Resuscitation 2016;105:79-84

23 Phattraprayoon N, Tangamornsuksan W, Ungtrakul T. Outcomes of endotracheal suctioning in non-vigorous neonates born 
through meconium-stained amniotic fluid: a systematic review and meta-analysis. Arch Dis Child Fetal Neonatal Ed 2021;106 (01):31-38

24 Selcuk AA. A guide for systematic reviews: PRISMA. Turk Arch Otorhinolaryngol 2019;57(01):57-58

25 Aslam S, Emmanuel P. Formulating a researchable question: A critical step for facilitating good clinical research. Indian J Sex Transm Dis AIDS 2010;31(01):47-50

26 Higgins JPT, Thomas J, Chandler J, et al. Cochrane Handbook for Systematic Reviews of Interventions Version 6.1. London: Cochrane Collaboration; 2020

27 Lo CK, Mertz D, Loeb M. Newcastle-Ottawa scale: comparing reviewers' to authors' assessments. BMC Med Res Methodol 2014; $14: 45$

28 Daga SR, Dave K, Mehta V, Pai V. Tracheal suction in meconium stained infants: a randomized controlled study. J Trop Pediatr 1994;40(04):198-200

29 Ting P, Brady JP. Tracheal suction in meconium aspiration. Am J Obstet Gynecol 1975;122(06):767-771

30 Velaphi S, Vidyasagar D. The pros and cons of suctioning at the perineum (intrapartum) and post-delivery with and without meconium. Semin Fetal Neonatal Med 2008;13(06):375-382
31 Clark DA, Nieman GF, Thompson JE, Paskanik AM, Rokhar JE, Bredenberg CE. Surfactant displacement by meconium free fatty acids: an alternative explanation for atelectasis in meconium aspiration syndrome. J Pediatr 1987;110(05):765-770

32 Tyler DC, Murphy J, Cheney FW. Mechanical and chemical damage to lung tissue caused by meconium aspiration. Pediatrics 1978;62 (04):454-459

33 Anand V, Basu S, Yadav SS, Narayan G, Bhatia BD, Kumar A. Activation of toll-like receptors in meconium aspiration syndrome. J Perinatol Official J Calif Perinatal Assoc 2018;38(02): 137-141

34 Chiruvolu A, Miklis KK, Chen E, Petrey B, Desai S. Delivery room management of meconium-stained newborns and respiratory support. Pediatrics 2018;142(06):e20181485

35 Vain NE, Szyld EG, Prudent LM, Wiswell TE, Aguilar AM, Vivas NI. Oropharyngeal and nasopharyngeal suctioning of meconiumstained neonates before delivery of their shoulders: multicentre, randomised controlled trial. Lancet 2004;364(9434):597-602

36 Kattwinkel J, Perlman JM, Aziz K, et al. Part 15: neonatal resuscitation: 2010 American Heart Association Guidelines for Cardiopulmonary Resuscitation and Emergency Cardiovascular Care. Circulation 2010;122(18, Suppl 3):S909-S919 